Voix et Images

volxetimages

\title{
Vers une définition du conte écrit au Québec : le récit court de type exemplum
}

\section{Jeanne Demers et Michelle Desjarlais-Konstantinov}

Volume 2, numéro 1, septembre 1976

Fernand Leduc

URI : https://id.erudit.org/iderudit/200025ar

DOI : https://doi.org/10.7202/200025ar

Aller au sommaire du numéro

Éditeur(s)

Les Presses de l'Université du Québec

\section{ISSN}

0318-9201 (imprimé)

1705-933X (numérique)

Découvrir la revue

\section{Citer cet article}

Demers, J. \& Desjarlais-Konstantinov, M. (1976). Vers une définition du conte écrit au Québec : le récit court de type exemplum. Voix et Images, 2(1), 110-118. https://doi.org/10.7202/200025ar d'utilisation que vous pouvez consulter en ligne. 


\section{Vers une définition du conte écrit au Québec: le récit court de type exemplum ${ }^{1}$}

Une morale nue apporte de l'ennui. Le conte fait passer le précepte avec lui.

La Fontaine

Qui, au Québec, ayant atteint un certain âge, ne se souvient de la célèbre réplique du petit catéchisme à la question portant sur l'ivrognerie: "L'ivrognerie [...] rend l'homme semblable à la bête et souvent le fait mourir." Et qui, parmi les amateurs de contes écrits, ne connaît le "Tom Caribou " de Louis Fréchette ${ }^{2}$ ? À première vue, il peut sembler étonnant que, dans une recherche portant sur le conte écrit soit tenté le rapprochement de cette réplique et d'un texte comme "Tom Caribou». Pourtant, entre le récit minimal, latent dans la sentence que constitue la réponse de catéchisme et celui, élaboré, que Fréchette prête à son conteur Jos Violon et qu'il insère dans le cadre d'une "veillée" à laquelle participe le narrateur, se situe toute une série de récits courts de type exemplum, qu'il nous a semblé utile d'explorer afin d'en venir à mieux délimiter la frontière qui marque la distance de cette sorte de récits au conte proprement dit et à la nouvelle ${ }^{3}$. Dans l'optique en effet d'une typologie du conte écrit québécois, typologie qui prétend ne pas exclure la thématique - au moins en autant qu'elle-est appelée par les formes - qui souhaite aussi déboucher éventuellement sur une meilleure connaissance de la société, il est indispensable de comprendre les mécanismes permettant le fonctionnement des "genres" voisins. Une définition (au sens du mot en télévision) du conte écrit et plus particulièrement du conte écrit québécois au XIX ${ }^{e}$ siècle, ne sera possible qu'à cette condition.

Au cours d'une première étape, nous avons procédé à une coupe dans le corpus, ne retenant que des textes traitant d'intempérance/tempérance, avec l'avantage certain de mettre d'autant mieux en évidence les formes qu'une sourdine se trouvait appliquée automatiquement au thème principal dont les variations, de récit à récit, ne pouvaient plus se faire 
qu'au plan du paradigme. Très rapidement des constantes se sont dégagées qu'il nous faudra examiner de plus près. Voyons d'abord les problèmes qu'a posés et que pose encore l'inventaire de ces textes, inventaire dont l'exhaustivité n'est cependant pas indispensable à notre projet, l'expérience nous ayant vite convaincues qu'un bon échantillonnage suffit amplement puisqu'ils se ressemblent de façon souvent affligeante, se pillant aussi volontiers les uns les autres.

L'exhaustivité d'un tel corpus serait-elle seulement possible d'ailleurs? On en doute dès que l'on est amené à constater l'ampleur du phénomène: d'innombrables ouvrages, de types fort divers, contiennent des récits courts dont le but avoué est l'enseignement ${ }^{4}$. II fallait d'une part les identifier, d'autre part recenser les bibliothèques où ils se trouvent. Les bibliothèques d'abord: les bibliothèques publiques ainsi que les collections d'archives qui relèvent directement des gouvernements, soit la Bibliothèque nationale du Canada, celle du Québec, les diverses bibliothèques municipales; les centres de recherche et de documentation des universités, certains musées privés subventionnés ou non par le ministère des Affaires culturelles du Québec, comme la Maison Saint-Gabriel, à Pointe-Saint-Charles, ou la Résidence Taschereau, à Sainte-Marie de Beauce.

$A$ ces bibliothèques et musées, s'ajoutent les bibliothèques et fonds d'archives des séminaires, scolasticats, juvénats et maisons de novices, maisons de retraite, patronnages, orphelinats, maisons de correction; enfin, les bibliothèques des palais épiscopaux, des presbytères et des cercles paroissiaux ont également accumulé des quantités importantes de volumes susceptibles de contenir des exempla, volumes parfois mal protégés, hélas: ainsi avant la démolition de l'ancien presbytère de Louiseville, on a demandé que soient mis de côté les "livres intéressants" avant d'enfouir “le reste » sous les décombres. «Le reste» étant, bien entendu, des ouvrages du type de ceux qui nous intéressent présentement.

Cette liste encore ouverte d'avenues à explorer - il serait possible d'y ajouter les manuels scolaires - était relativement facile à établir. Les difficultés commençaient ensuite. L'index des sujets dans les fichiers consultés faisait éclater en étoile le réseau des possibles: annales, bulletins, journaux, messagers, semaines religieuses, tracts et autres périodiques s'ajoutaient à la longue liste des textes expressément didactiques au chapitre de la doctrine et de la morale, opuscules, recueils, traités portant en intitulé les mots abrégé, allocutions, cathéchisme, carêmes, causeries, chroniques, clefs, conférences religieuses, congrès eucharistiques, controverses, dévotions, dialogues, discours, éloges, esquisses, exercices, guide, homélie, instructions, neuvaines prêchées, notes, notices, panégyriques, paroissien, petite histoire, règlement, rituel, sermon, statuts, témoignages, vie... et la liste n'est pas terminée!

La prédication dominicale aurait dû nous fournir des matériaux, mais très peu de prônes et de sermons ordinaires semblent avoir été publiés. Quant aux sermons manuscrits, on peut rarement en trouver sinon de très 
anciens. La coutume ecclésiastique voudrait que l'exécuteur testamentaire d'un membre du clergé cherche d'abord les sermons dès qu'il commence l'inventaire des biens personnels de celui-ci. Pour les détruire. Prudence, déplorable en ce qui nous concerne. Grâce au travail de Monsieur Louis Rousseau du module des Sciences religieuses de l'Université du Québec à Montréal, la Bibliothèque nationale du Québec conserve toutefois sur microfilms une grande quantité de sermons prêchés en particulier à l'église Notre-Dame de Montréal. Nous n'avons pas encore pu explorer cette documentation.

Nous avons par contre dépouilié la section des ouvrages religieux de la collection Melzac, confiée au Service des collections particulières des bibliothèques, à l'Université de Montréal. Il y a sans doute dans d'autres collections privées des découvertes intéressantes à faire.

L'exemplum n'est pas toujours facile à reconnaître. Si dans les meilleurs des cas, il se présente isolé sur la page par la disposition typographique, il surgit souvent dans le texte, quelquefois sans doute à l'insu même du scripteur qui éprouve comme un besoin pédagogique d'étayer concrètement une argumentation serrée. L'exemple est-il isolé ? II peut se trouver ou non précédé du(des) $\operatorname{mot}(\mathrm{s})$ “exemple», “autre exemple», "autres exemples"; "autres exemples» annonçant évidemment une série de récits habituellement très brefs, quelques lignes tout au plus.

A l'occasion, l'exemple va jusqu'à se réduire à une sorte de degré zéro, comme dans ce commentaire de Chiniquy:

Je vous citerais bien d'autres traits dont mes livres sont remplis des funestes effets de la boisson; mais ce serait à ne jamais finir, et je serais véritablement embarrassé sur le choix ${ }^{5}$.

Degré zéro d'existence dû, paradoxalement, au fait que l'exemple pourrait être multiplié à l'infini. "En voulez-vous des exemples?", de s'exclamer l'abbé Mailloux... «Tant que vous en voudrez; j'en ai pour remplir un gros volume ${ }^{6}$."

Le classement provisoire que nous avons tenté des diverses formes empruntées par le récit court de type exemplum au XIXe siècle québécois, révèle seulement la partie émergée de l'iceberg. II montre déjà cependant à quel point l'exemple diffère du conte et de la nouvelle; l'exemple et avec lui tous les récits qui, bien que s'annonçant souvent comme conte ou nouvelle, ne s'éloignent guère du modèle qu'il suppose et ressemblent à s'y méprendre aux "anecdotes" ou «illustrations» de certains sermonnaires.

Du texte systématique en effet qui forme en quelque sorte le noyau, le principe de l'exemplum - la réplique de cathéchisme rappelée plus haut ou l'exemple latent "L'alcool ne nourrit pas; il abrutit et tue ${ }^{7}$ " - au texte narratif (exemple minimal), que celui-ci soit allusif, un mot comme 
"Noé", par exemple, ou composé d'une ou de plusieurs propositions", des modifications profondes ont lieu. Et nous sommes pourtant encore bien éloignés du conte ou de la nouvelle! II se passe ce que Lessing, cité par Stierle9, a magnifiquement pointé du doigt dans une séquence narrative - pour que le médium se fasse vraiment message sans doute - à propos de la fable actualisée dans le récit minimal «Le loup dévora le renard" et provenant de la sentence "Le plus faible est la proie désignée du plus fort» après un certain nombre de transformations dont l'avant-dernier état peut se lire ainsi : "Le loup dévore le renard."

Du principe moral le plus large donc, à l'histoire particulière. En outre, à partir du moment que le geste d'agression du plus fort envers le plus faible a été identifié - ici le fait de dévorer -, à partir du moment également que le présent s'est modifié en aoriste, il ne peut s'agir de n'importe quel loup ni de n'importe quel renard. Nous pourrions même nommer l'un Ysengrin et l'autre, le goupil, Renart justement... Les situer aussi dans l'espace et dans le temps... Mais n'anticipons pas.

Demandons-nous plutôt à quelles tensions le récit minimal contenu en puissance dans «L'alcool ne nourrit pas; il abrutit et tue se trouve exposé pour devenir soit exemplum, soit conte ou nouvelle. La première, celle qui oriente tout l'avenir du texte, c'est indiscutablement son projet. N'est-ce pas ce dernier qui organise et actualise le schéma narratif? Or à quoi prétend le discours qui annonce le récit dans le cas d'exemples déployés? Et leur contexte extra-linguistique - types d'ouvrages dans lesquels ils paraissent, occasion de leur publication, etc. - quelle intention projette-t-il sur eux sinon celle de convaincre le lecteur de ne pas boire?

Lisons le passage qui précède immédiatement l'un de ces exemples: l'on verra qu'aucune ambiguïté ne demeure quant à ses intentions:

Jeunes gens qui ne craignez point de boire ces funestes liquides, qui prenez le chemin de perdre et votre raison et votre intelligence et votre cœur, daignez lire ce que je vais écrire pour vous. Frère coupable, immensément coupable, qui vous êtes accoutumé à vous livrer a cette passion hideuse de l'ivrognerie, ne détournez point vos regards du spectacle que je vais vous présenter. Vous tous que la sainte tempérance de la croix a arrachés à cette affreuse passion, vous surtout qu'elle a préservés de vous en laisser dominer, venez étudier, contempler l'homme, la plus belle créature de la terre sous les coups de l'affreuse passion de l'ivresse. Venez tous vous instruire, et, en vous instruisant, concevoir une haine sans borne [pour ce qui] conduit si souvent, et si fatalement, à l'intempérance et aux désordres qui en sont les suites funestes ${ }^{10}$.

Convaincre le lecteur de ne pas boire. La contrainte d'un tel but ne peut que limiter le récit, le retenir d'une certaine manière sur la voie de ses possibles, dans sa grammaire narrative comme dans sa grammaire textuelle, d'où, d'ailleurs, la brièveté relative de l'exemplum. Tout au plus le récit pourra-t-il se déployer bien modestement au plan de l'événement. II se présentera alors comme l'exemple suivant: 
Quelques écoliers, en vacances, jeunes gens de 16 à 17 ans, s'étaient réunis chez un de leurs confrères. Ils étaient tous de familles très respectables; ils n'auraient jamais voulu donner une mauvaise opinion d'eux-mêmes; ils voulaient se tenir comme il faut.

Cependant passer quelques heures ensemble sans prendre de boisson, il ne fallait pas y songer; aussi sans être ivres, furent-ils bientôt gais comme pinsons. C..., présumant trop de ses forces, avait mesuré le coup trop fort. II s'aperçut bientôt que la terre tournait plus que d'ordinaire; les objets se multipliaient, sous ses yeux fatigués d'une manière alarmante; il fit pourtant bonne contenance jusqu'à l'heure du "souper. C'est là que le ridicule et la confusion l'attendaient. On lui verse une tasse de thé; mais, soudain, son imagination un peu échauffée lui laisse apercevoir trois soucoupes... Que faire?... II voudrait bien ne pas se compromettre en faisant une sottise... II se croit obligé de se servir d'une soucoupe... Laquelle choisir?... Impossible de me tromper en prenant celle du milieu, se dit-il...; et voilà $X .$. qui verse lentement son thé dans la soucoupe du milieu, c'est-à-dire... sur la nappe! La honte le dégrisa aussitôt, et il promit bien de ne plus se laisser prendre ${ }^{11}$.

Ou encore, par le biais du discours rapporté, de la description, du portrait, tirés constamment au discours, il se dramatisera au profit immédiat de la leçon:

... Instruisons-nous, en voyant ce qui se passe dans ce bouge de l'infamie. Le vaincu de Satan s'approche de la barre [sic]. Le commis de Satan se hâte de lui verser un verre. II I'avale avec l'avidité fébrile d'un frénétique. Et puis, contemplez-le: semblable à la bête féroce qui a bu du sang, il se lèche les lèvres, pour ne rien perdre de cette affreuse potion. Le feu qu'il vient de jeter dans ses entrailles a allumé la passion de l'ivresse. Encore un verre, s'écrie-t-il! encore un verre! Et le second verre est immédiatement versé! Cette fois, regardez les yeux de l'intempérant. Ils brillent comme ceux du hibou au milieu des ténèbres de la nuit. Ce verre est saisi avec une compulsion nerveuse qui menace de briser le fragile vase qui contient l'ivresse. II l'a avalé ! oui, il l'a encore avalé, et c'eut été déjà trop d'un seul verre!

Les deux verres du liquide vont produire leur effet. Son intelligence se trouble, sa raison disparaît... etc. ${ }^{12}$.

Quant à la fin de l'histoire, elle se trouve comme préinscrite avant que celle-ci ne se déroule. Pas question en effet d'imaginer un ivrogne heureux ou rendant les siens heureux.

Dans l'exemplum, le récit doit impressionner, frapper les imaginations pour gagner les cœurs et les volontés. II n'a surtout pas à plaire. Du moins pas d'abord. Le plaisir du texte, nécessairement subversif, risquerait d'égarer le lecteur hors du chemin étroit que lui trace l'exemplarité d'un fait dont l'unicité n'est que garante du général. II en va bien 
autrement pour le conte écrit et la nouvelle qui n'ont de raison d'être que s'ils plaisent et de réalité que dans un particulier n'ayant de liens avec le général que ceux qu'il veut bien avoir. Ainsi dans le conte de Paul Stevens, intitulé "les Trois diables ${ }^{13}$ ", la femme du meunier ne cesse de boire. Le narrateur qui constate le fait précise même "car, comme dit le proverbe: «qui a bu, boira ${ }^{14}$ ». Mais voilà qui montre la distance entre le conte écrit même exemplaire (la nouvelle) et l'exemple: le proverbe, ici, ne fonde pas l'histoire. II ne fait que coïncider avec elle. Son rôle - et signalons en passant qu'il relève de la grammaire textuelle tout comme le «II y avait une fois un cordonnier..." de la phrase initiale - en est un de complicité, de connivence entre le narrateur et le lecteur qui font partie du même contexte culturel. Et si ce n'était pas le cas, il serait alors simplement explicatif.

Quant à la morale, explicite, elle se trouve «récupérée» par les qualités stylistiques du texte, contrairement à ce qui aurait eu lieu dans l'exemplum:

Si nous vivons bien, chers et bons lecteurs, nous aurons un jour l'avantage et le bonheur de faire connaissance là-haut avec le brave Richard, et j'ai l'intime conviction qu'il vous garantira de point en point l'exactitude de cette étonnante et véridique histoire que j'aurais voulu pouvoir vous raconter mieux, et surtout avec ces gestes inimitables dont mon ami Richard semble avoir seul le secret ${ }^{15}$.

Une telle morale révèle une autre caractéristique du conte (de la nouvelle) qui le(la) rend bien différent(e) de l'exemple: son autonomie. Par autonomie, nous entendons sa possibilité d'exister, sans mutilation, indépendamment de son contexte immédiat. II peut arriver que cette autonomie soit relative dans un recueil de contes (de nouvelles) à organisation particulièrement serrée. Les relations de conte à conte (de nouvelle à nouvelle) ne seraient toutefois pas du même ordre que celles établies par le discours et le contexte extra-linguistique qui provoquent l'exemplum : elles seraient syntagmatiques et non paradigmatiques.

Ne nous y trompons pas toutefois. L'autonomie d'un récit ne suffit pas à faire un conte ou une nouvelle d'un exemple ou d'un récit qui aurait presque toutes les caractéristiques de l'exemple. Aussi existe-t-il des textes qui constituent de véritables cas frontières exemples/contes (nouvelles); “l'Enfant perdu» de J.F. Morissette ${ }^{16}$ en est un bon échantillon. II s'agit, en quelques pages, du récit désespérément terne de la mort d'un enfant, mort provoquée par l'ivrognerie du père. Le texte est autonome.

Il fait partie d'un recueil qui accumule au hasard, le sous-titre le dit, «des nouvelles, récits et légendes». Son projet n'est pas de convaincre des dangers de la boisson, puisqu'il s'inscrit - et cette fois c'est le titre qui nous l'apprend - «au coin du feu», lieu privilégié du conte, comme chacun sait. De plus le texte ne comporte aucune morale explicite. Et si la fin en contient une, celle-ci reste implicite:

Alfred Lambert fut tellement frappé par la mort de son fils qu'il n'osât [sic] plus prendre une goutte de boisson ${ }^{16}$. 
“L'Enfant perdu " s'éloigne-t-il pour autant de l'exemplum? Certains éléments nous paraissent lui manquer pour qu'il accède tout à fait à la nouvelle qu'il prétend être. Le plaisir du texte d'abord, qu'il est toutefois, nous le reconnaissons difficile à codifier. Ce plaisir d'ailleurs ne représente-t-il pas toujours le grand inconnu, en littérature? Le fait ensuite que le général y demeure fortement latent dans le particulier. Le personnage principal peut bien se nommer en effet Alfred Lambert, et sa femme, Alvina Lafortune. Le narrateur peut aussi donner leurs antécédents et même la date et l'heure de l'événement:

La veille du premier de l'an 1878, vers 7 heures du soir ${ }^{17}$.

Ces seules précisions n'arrivent pas à individualiser les uns et à particulariser les autres, condition pourtant essentielle à l'existence de la nouvelle.

À quoi sert au lecteur de savoir de Lambert que

Dès l'âge de 15 ans son père [sic] était obligé d'aller le chercher dans les maisons où l'on débitait de la boisson sans licence [sic] ${ }^{18}$,

si, tout le long du texte, c'est l'ivrognerie qui constitue le véritable meneur de jeu, comme en font foi les nombreuses occurrences des mots «ivrogne", «ivre», "vice", «boire", etc. ? Si c'est cette ivrognerie qui se révèle la cause profonde de l'écart, d'ailleurs simpliste, entre la mauvaise conduite de Lambert - celle aussi de son double dans le mal, l'oncle d'Alvina - et la bonté de ceux qui l'entourent:

Les bons conseils de son père qui était un parfait honnête homme, ni les pleurs de sa bonne mère ne réussirent à le corriger. II continuait à boire et devenait de plus en plus ivrogne ${ }^{19}$.

Si enfin, l'argument du récit, parce que maladroitement actualisé, se rapproche plus, pour adapter à notre propos la fable de Lessing, de la constatation générale “l'ivrogne cause le malheur des siens", tirée de la sentence "L'ivrognerie [...] abrutit et tue", que du récit minimal "l'ivrogne cause le malheur des siens"?

Le coup de sonde que nous avons jeté du côté de l'exemple ne prétend évidemment pas épuiser la question. Nous croyons cependant qu'il nous a permis une première analyse déjà précieuse en ce qui concerne une éventuelle typologie du récit court québécois. Le passage en effet du texte systématique au texte narratif ne se produit pas sans transformations tant au plan des signaux linguistiques qu'à ceux de l'énoncé et des thèmes. II en va de même du texte narratif minimal au conte écrit ou à la nouvelle et, bien entendu, entre celle-ci et le conte écrit qui diffère également dans ses manifestations. L'analyse des textes devrait nous amener à 
découvrir les lieux et les modalités de ces transformations pour éventuellement nous conduire à un classement qui, au lieu de se faire à partir de rubriques sous lesquelles il faudrait coûte que coûte forćer les diverses réalisations du récit court québécois, serait établi par un système vertical à créneaux révélant clairement, d'une part les dominantes de chaque type et d'autre part, comment chaque actualisation s'inscrit par rapport à ces dominantes. Mais cela fera l'objet d'un autre chapitre...

Jeanne Demers

Université de Montréal

et

Michelle Desjarlais-Konstantinov

CEGEP Champlain

1. Cette étude du récit court de type exemplum qui s'inscrit dans le cadre d'une recherche sur le conte écrit québécois, le projet DEVIN (Jeanne Demers et Lise Gauvin), subventionné par le Conseil des arts, a fait l'objet d'une communication à l'ACFAS, le jeudi 13 mai 1976, à Sherbrooke.

2. Contes de Jos Violon, présentation de Victor-Lévy Beaulieu, Montréal, L'Aurore, 1974 , p. $35-50$.

3. Notre propos n'étant pas pour le moment de distinguer ces deux types de récits courts, nous les mettrons tous deux sur le même pied, bien qu'ils présentent des différences importantes.

4. Définition un peu simple, nous le savons. Elle n'est que provisoire toutefois et nous paraît mieux convenir à notre propos que les nombreuses autres qui ont déjà été proposées. Voir à ce sujet: J.-Th. Welter, l'Exemplum dans la littérature religieuse et didactique du moyen âge, Paris et Toulouse, Occitania, 1927, p. 1 et 2.

5. Manuel ou règlement de la Société de tempérance/dédié à la jeunesse canadienne, Québec, Stanislas Drapeau, 1844, p. 108.

6. L'ivrognerie est l'cuuvre du démon mais la sainte tempérance de la Croix est I'œuvre de Dieu, Québec, Augustin Côté et Cie, 1867, p. 82.

7. Constant Doyon, o.p., Ia Lutte antialcoolique/Simples articles, Québec, Action sociale, 1911, p. 20.

8. Cf. l'exemple suivant tiré de L'ivrognerie est l'œuvre du démon mais la sainte tempérance de la Croix est l'œuvre de Dieu, p. 44-45: "Balthasar, dejà plein de vin, fait apporter sur la table d'un festin, les vases d'or et d'argent que Nabuchodonosor, son pere, avait enlevé du temple de Dieu, à Jérusalem. Pendant qu'il les fait servir à ses orgies et à celles de ses concubines et des grands de sa cour, il aperçoit une main qui écrit, sur le mur de la salle du festin, sa sentence de mort, et il est tué la même nuit. »

9. Dans "l'Histoire comme Exemple, l'Exemple comme Histoire", Poétique $n^{\circ} 10,1972$, p. 180-181. L'ouvrage de Lessing dont il est question s'intitule en traduction, Réflexions sur la fable. Quant à l'article mëme de Stierle, il a profondément marqué la présente réflexion.

10. Chiniquy, op. cit., p. 51.

11. Charles Larocque, abbé, Guerre à l'intempérance/Lisez et méditez, $2^{\mathrm{e}}$ éd., Montréal, J. Chapleau et fils, 1887, p. 30-31.

12. Alexis Mailloux, prêtre, op. cit., p. 51 et suiv.

13. Dans Contes populaires, Ottawa, G.E. Desbarats, 1867, p. 51-65.

14. Ibid., p. 58.

15. Ibid., p. 65 . Ajoutons que certains des thèmes du conte écrit "les Trois diables" appartiennent à la thématique internationale du conte oral: le diable, les trois souhaits, le violon magique, etc. 
118 voix et images

16. Montréal, Piché Frères, 1883, p. 33-40.

17. Ibid., p. 33.

18. $I d$.

19. Id. 